\title{
Simultaneous Siting and Sizing of Distribution Centers on a Plane
}

\author{
Simin Huang*, Rajan Batta** and Rakesh Nagi**1 \\ *Department of Industrial Engineering, Tsinghua University, Beijing 100084, \\ People's Republic of China. \\ ** Department of Industrial Engineering, 342 Bell Hall, \\ University at Buffalo (SUNY), Buffalo, NY 14260, USA.
}

Revised: September 2005

December 2004

\footnotetext{
${ }^{1}$ For Correspondence: Email: nagi@buffalo.edu, Tel: +1-716-645-2357x2103, Fax: +1-716-645-3302.
} 


\begin{abstract}
The benefits of simultaneous consideration of siting and sizing of distribution centers have been well acknowledged in supply chain design. Most formulations assume that the potential DC sites are known and the decision on location is to select sites from the finite potential DC sites. However, the quality of this discrete version problem depends on the selection of potential DC sites. In this paper we present a planar version of the problem, which assumes that there is no a priori knowledge of DC sites and DCs can be located anywhere in the plane. The goal of the problem is to simultaneously find locations and sizing of DC sites. The solution of the planar problem provides a lower bound for the discrete problem. The objective of the problem is to minimize the total of inbound and outbound transportation costs and distribution center construction costs - which include its fixed charge cost and concave sizing cost. The problem is initially formulated as a nonlinear programming model. We then reformulate it as a set covering problem after establishing certain key properties. A greedy drop heuristic and a column generation heuristic are developed to solve the problem. Computational experiments are provided.
\end{abstract}

Keywords: Distribution center sizing; Distribution center location; Supply chain design. 


\section{Introduction}

This paper considers an integrated distribution center (DC) planar location and sizing problem in which products are shipped from plants to distribution centers (DCs) and then delivered to retailers. The objective of the problem is to minimize the total inbound and outbound transportation costs and distribution center construction costs - which include its fixed charge cost and concave sizing cost. Most formulations assume that the potential DC sites are known and the decision on location is to select sites from the finite set of potential DC sites. However, the quality of this discrete version problem depends on the selection of potential DC sites. In this paper we assume that there is no a priori knowledge of DC sites and DCs can be located anywhere on the plane. Therefore, the decision on location in this problem is not to select but to generate DC sites. This version of the problem provides a lower bound for the discrete problem. When the gap between the solutions of the discrete and planar models is significant, it suggests that more potential DC sites should be added in the candidate set of the discrete formulation. Another motivation of this paper is based on the view that the design of such a system can be improved by simultaneous consideration of location and sizing because of the trade-off between transportation costs and sizing costs. However, the size issue in the planar location literature is neglected. In fact, Krarup and Pruzan (1990) (page 41) point out that in most formulations reported in the literature, the notion of facility size is essentially ignored and facilities are implicitly assumed to be points in space as are the sites where they may be located.

The most related problem developed in the location literature is the planar location-

allocation problem, which was initiated in papers by Cooper (1963) and Cooper (1964). The 
problem is to locate a set of $p$ new facilities in a plane and assign a set of $m$ existing facilities to the new facilities in order to minimize total weighted distances. The main difficulty in solving this problem arises from the fact that its objective function is neither convex nor concave (see Cooper (1967)), and it is not differentiable everywhere. In general, it contains a large number of local minima (see Eilon, Watson-Gandy, and Christofides (1971)). Therefore, most models in this area assume that facilities are uncapacitated. Very few planar location models consider the sizing issue. As far as we know, the only exception is the paper by Brimberg and Love (1994). They consider a location-allocation problem with economies of scale and present heuristic approaches to solve small-size problems. An overview of applications of location-allocation problems is given in Hodgson, Rosing, and Shmulevitz (1993).

The other related problem is the facility sizing problem. It seeks to find a facility size which allows a required service level to be attained and/or minimizes total costs. Facilities can be broadly defined as buildings where people, material, and machines come together for a stated purpose - typically to make a tangible product or provide a service (see Heragu (1997)). Therefore, it is natural to classify the facility sizing problem based on its space requirement, server number, inventory size, or machine capacity (or types). The warehouse space requirement problem can be found in Sung and Han (1992), White and Francis (1971), Lowe, Francis, and Reinhardt (1979) and Jucker, Carlson, and Kropp (1982). The server allocation of queueing facilities has been studied in Brimberg, Mehrez, and Wesolowsky (1997) and Brimberg and Mehrez (1997). For warehouse inventory sizing problem, Cormier and Gunn (1996b) and Cormier and Gunn (1996a) coordinate warehouse size and inventory policy under the assumption of constant product demand. In the case of deterministic facility capacity, Lee (1991) and Mazzola and Neebe (1999) study a multi-product capacitated 
facility location problem with a choice of facility type. All of these models assume that either facility locations are fixed or there is a priori knowledge of facility locations. Also, sizing is a major issue in Huff Gravity models. A recent reference where sizing are optimized is Aboolian, Berman, and Krass (2004).

This paper is organized as follows: Section 2 presents a mathematical formulation of the problem and establishes properties of the model. Section 3 reformulates the model as a set covering problem. A greedy drop heuristic and a column generation heuristic are developed to solve it. Section 4 reports on computational results for both algorithms. Finally, Section 5 provides a summary and directions for future work.

\section{Formulation}

We are given a set of plant nodes, $I=\{1,2, \cdots, m\}$, and a set of retailer nodes, $J=$ $\{1,2, \cdots, n\}$. Let $(i, j)$ denote a flow from plant $i$ to retailer $j$ and $f_{i j}$ its flow amount, $\forall i \in I$ and $j \in J$. Let $k \in K=\{1,2, \cdots, p\}$ be a DC index.

We assume that the transportation costs are determined by the distances of the shortest paths between nodes. Let $Y_{k}$ be the location decision variable of $\mathrm{DC} k$ and $d\left(i, Y_{k}\right)$ be the shortest distance between plant node $i$ and DC $k$, where $d(\cdot)$ is a distance measure. Let $x_{i j k}$ be the fraction of flow amount $f_{i j}$ that passes through DC $k$. Then, the transportation cost can be expressed as

$$
\sum_{i \in I} \sum_{j \in J} \sum_{k \in K} f_{i j}\left(d\left(i, Y_{k}\right)+d\left(Y_{k}, j\right)\right) x_{i j k} .
$$

Generally speaking, DC sizing cost is based on its space requirement, server number, inventory size, or machine capacity (or types), which should be determined by the maximum 
flow amount that can go through the DC. Let $c_{k}$ be the sizing variable of DC $k$. We assume that the sizing cost function of DC $k, f_{k}(\boldsymbol{c})$, includes its fixed charge cost and concave sizing cost. It takes the form as follows:

$$
F \times I\left(c_{k}\right)+h\left(c_{k}\right), \forall k \in K
$$

where $F$ is a constant which denotes the fixed charge cost of a DC, $I\left(c_{k}\right)$ is an indicator function with $I(0)=0$ and $I\left(c_{k}>0\right)=1$, and $h\left(c_{k}\right)$ is an increasing concave function of $c_{k}$ and $h(0)=0$.

The objective of the simultaneous DC siting and sizing problem is to minimize total of inbound and outbound transportation costs and distribution center sizing costs. Then, the problem can be formulated as follows:

$$
\begin{aligned}
& \text { (P) } \quad \min _{\boldsymbol{x}, \boldsymbol{y}} Z^{P}(\boldsymbol{c}, \boldsymbol{x}, \boldsymbol{y})=\sum_{i \in I} \sum_{j \in J} \sum_{k \in K} f_{i j}\left(d\left(i, Y_{k}\right)+d\left(Y_{k}, j\right)\right) x_{i j k} \\
& +\sum_{k \in K}\left(F \times I\left(c_{k}\right)+h\left(c_{k}\right)\right) \\
& \text { subject to } \quad \sum_{k \in K} x_{i j k}=1, \quad \forall i \in I, j \in J \text {, } \\
& \sum_{i \in I} \sum_{j \in J} f_{i j} x_{i j k} \leq c_{k}, \quad \forall k \in K, \\
& x_{i j k}, c_{k} \geq 0, Y_{k} \in R^{2} \quad \forall i \in I, j \in J, k \in K .
\end{aligned}
$$

The objective function (1) minimizes the total transportation cost and DC sizing cost. Constraint (2) stipulates that the flows are only transported through DCs. Constraint (3) is a capacity constraint. Constraints (4) are non-negativity constraints.

The major difficulty in solving this problem is due to the form of the objective function, which is neither convex nor concave. We establish some properties with the hope of finding 
an effective solution procedure for Problem $(P)$. For simplicity, we develop the properties and a heuristic algorithm under the Manhattan distance. However, they can be extended to the general polyhedral gauge case (see Huang, Batta, Klamroth, and Nagi (2005)).

In this paper, we consider the grid construction for the Manhattan distance as follows: Consider the smallest rectangle (bounding rectangle) that encloses all plants and retailers and whose sides are parallel to the $x$ and $y$ axes. Within this bounding rectangle, the grid is formed by lines parallel to the $x$ and $y$ axes through all plant and retailer nodes. A grid point is an intersection point of any two lines.

Property 1. There exists at least one optimal solution of Problem $(P)$ with the Manhattan distance for which each DC is located on a grid point.

Proof: If all $Y_{k}^{*}$ s are located at grid points, then we already obtain the property.

Suppose that the assertion is not true and let $\left(\boldsymbol{c}^{*}, \boldsymbol{x}^{*}, \boldsymbol{y}^{*}\right)$ be an optimal solution for Problem $(P)$. If all $Y_{k}^{*} \mathrm{~s}$ are located at grid points, then we already obtain the property. If at least one of them is not located at a grid point, without loss generality, we assume that $Y_{1}^{*}$ is not a grid point and located inside the rectangle $A B C D$ (see Figure 1); here $A, B, C$, and $D$ are grid points and there are no grid points in the interior of rectangle $A B C D$. Let $R_{A}, R_{B}, R_{C}$, and $R_{D}$ be the northwestern region of node $A$, the northeastern region of node $B$, the southwestern region of node $C$, and the southeastern region of node $D$, respectively. We also assume that

$$
\begin{aligned}
& \sum_{i \in R_{A}} \sum_{j \in R_{C}} f_{i j} x_{i j 1}^{*}+\sum_{i \in R_{C}} \sum_{j \in R_{A}} f_{i j} x_{i j 1}^{*} \geq \sum_{i \in R_{B}} \sum_{j \in R_{D}} f_{i j} x_{i j 1}^{*}+\sum_{i \in R_{D}} \sum_{j \in R_{B}} f_{i j} x_{i j 1}^{*}, \\
& \sum_{i \in R_{A}} \sum_{j \in R_{B}} f_{i j} x_{i j 1}^{*}+\sum_{i \in R_{B}} \sum_{j \in R_{A}} f_{i j} x_{i j 1}^{*} \geq \sum_{i \in R_{C}} \sum_{j \in R_{D}} f_{i j} x_{i j 1}^{*}+\sum_{i \in R_{D}} \sum_{j \in R_{C}} f_{i j} x_{i j 1}^{*} .
\end{aligned}
$$

Let $\Delta_{1}$ be the horizontal distance between $Y_{1}^{*}$ and $A$ and $\Delta_{2}$ be the vertical distance 
between $Y_{1}^{*}$ and $A$. Now, if we move $Y_{1}^{*}$ to the grid point $A$ and all others remain unchanged, then the net improvement of the objective function value is

$$
\begin{gathered}
\Delta_{1}\left(\sum_{i \in R_{A}} \sum_{j \in R_{C}} f_{i j} x_{i j 1}^{*}+\sum_{i \in R_{C}} \sum_{j \in R_{A}} f_{i j} x_{i j 1}^{*}-\sum_{i \in R_{B}} \sum_{j \in R_{D}} f_{i j} x_{i j 1}^{*}+\sum_{i \in R_{D}} \sum_{j \in R_{B}} f_{i j} x_{i j 1}^{*}\right) \\
+\Delta_{2}\left(\sum_{i \in R_{A}} \sum_{j \in R_{B}} f_{i j} x_{i j 1}^{*}+\sum_{i \in R_{B}} \sum_{j \in R_{A}} f_{i j} x_{i j 1}^{*}-\sum_{i \in R_{C}} \sum_{j \in R_{D}} f_{i j} x_{i j 1}^{*}+\sum_{i \in R_{D}} \sum_{j \in R_{C}} f_{i j} x_{i j 1}^{*}\right) \geq 0 .
\end{gathered}
$$

Thus the objective function will not increase if we move $Y_{1}^{*}$ to a grid point. This procedure can be repeated for any other $Y_{k}^{*}$ which is not currently at a grid point. Thus a grid point solution of equal or superior value can be constructed from any non-grid point solution. The property follows.

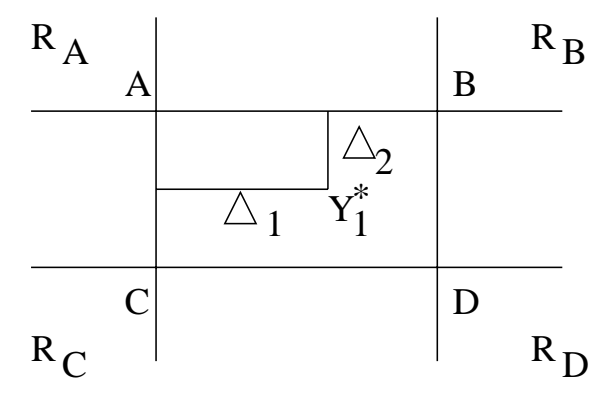

Figure 1: Proof of grid point optimality

Property 2. If $\left(\boldsymbol{c}^{*}, \boldsymbol{x}^{*}, \boldsymbol{y}^{*}\right)$ is an optimal solution for Problem $(P)$, then

$$
c_{k}^{*}=\sum_{i \in I} \sum_{j \in J} f_{i j} x_{i j k}^{*}, \forall k \in K
$$

Proof: Suppose that there exists an $l \in K$ such that $c_{l}^{*} \neq \sum_{i \in I} \sum_{j \in J} f_{i j} x_{i j l}^{*}$. According to constraint (3), $c_{l}^{*}>\sum_{i \in I} \sum_{j \in J} f_{i j} x_{i j l}^{*}$. Let $c_{l}^{\prime}=\sum_{i \in I} \sum_{j \in J} f_{i j} x_{i j l}^{*}$, then $h\left(c_{l}^{*}\right)>h\left(c_{l}^{\prime}\right)$ (because $h(\cdot)$ is an increasing function). Using $c_{l}^{\prime}$ to replace $c_{l}^{*}$ in the optimal solution, we can claim that the new solution has a lower objective function value. The result follows by contradiction. 
Thus, Problem $(P)$ is equivalent to the following problem:

$$
\begin{aligned}
& (E P) \quad \min _{\boldsymbol{c}, \boldsymbol{x}, \boldsymbol{y}} Z^{E P}(\boldsymbol{c}, \boldsymbol{x}, \boldsymbol{y})=\sum_{i \in I} \sum_{j \in J} \sum_{k \in K} f_{i j}\left(d\left(i, Y_{k}\right)+d\left(Y_{k}, j\right)\right) x_{i j k} \\
& +\sum_{k \in K}\left(F \times I\left(\sum_{i \in I} \sum_{j \in J} f_{i j} x_{i j k}\right)+h\left(\sum_{i \in I} \sum_{j \in J} f_{i j} x_{i j k}\right)\right) \\
& \text { subject to } \quad \sum_{k \in K} x_{i j k}=1, \quad \forall i \in I, j \in J, \\
& x_{i j k}, c_{k} \geq 0, Y_{k} \in R^{2} \quad \forall i \in I, j \in J, k \in K .
\end{aligned}
$$

Before we establish Property 3, we need to use the approach in Brimberg and Love (1994) to establish that the flows from plants to retailers can be assumed to be assigned to exactly one DC.

Lemma 1. If an optimal solution for Problem $(P)$ exists where a flow $(i, j)$ is assigned to two DCs $k$ and $l$, then we have

$$
f \frac{d h\left(c_{k}\right)}{d c_{k}}+f_{i j}\left(d\left(i, Y_{k}\right)+d\left(Y_{k}, j\right)\right)=f \frac{d h\left(c_{l}\right)}{d c_{l}}+f_{i j}\left(d\left(i, Y_{l}\right)+d\left(Y_{l}, j\right)\right)
$$

where $d h\left(c_{k}\right) / d c_{k}$ denotes the first derivative of the sizing cost function $h(\cdot)$ and $f=$ $\sum_{i \in I} \sum_{j \in J} f_{i j}$

Proof: If the statement is not true, then we assume without loss of generality that

$$
f \frac{d h\left(c_{k}\right)}{d c_{k}}+f_{i j}\left(d\left(i, Y_{k}\right)+d\left(Y_{k}, j\right)\right)<f \frac{d h\left(c_{l}\right)}{d c_{l}}+f_{i j}\left(d\left(i, Y_{l}\right)+d\left(Y_{l}, j\right)\right) .
$$

Note that $c_{k}=\sum_{i \in I} \sum_{j \in J} f_{i j} x_{i j k}$ in any optimal solution. If we perturb $x_{i j k}$ by a quantity $\delta>0$ and $x_{i j l}$ by $-\delta$, then $\sum_{i \in I} \sum_{j \in J} f_{i j}\left(x_{i j k}+\delta\right)=c_{k}+\delta f$. Hence, the net improvement of the objective function value equals (assuming that both connections $k$ and $l$ are still open after the perturbation)

$$
f_{i j}\left(d\left(i, Y_{k}\right)+d\left(Y_{k}, j\right)\right)\left(x_{i j k}+\delta\right)+h\left(c_{k}+\delta f\right)+f_{i j}\left(d\left(i, Y_{l}\right)+d\left(Y_{l}, j\right)\right)\left(x_{i j l}-\delta\right)+h\left(c_{l}-\delta f\right)
$$




$$
\begin{gathered}
-\left(f_{i j}\left(d\left(i, Y_{k}\right)+d\left(Y_{k}, j\right)\right) x_{i j k}+h\left(c_{k}\right)+f_{i j}\left(d\left(i, Y_{l}\right)+d\left(Y_{l}, j\right)\right) x_{i j l}+h\left(c_{l}\right)\right) \\
=\delta\left(f \frac{h\left(c_{k}+\delta f\right)-h\left(c_{k}\right)}{\delta f}+f_{i j}\left(d\left(i, Y_{k}\right)+d\left(Y_{k}, j\right)\right)-\left(f \frac{h\left(c_{l}+\delta f\right)-h\left(c_{l}\right)}{\delta f}+f_{i j}\left(d\left(i, Y_{l}\right)+d\left(Y_{l}, j\right)\right)\right)\right) .
\end{gathered}
$$

Since $d h\left(c_{k}\right) / d c_{k}=\lim _{\delta f \rightarrow 0} \frac{h\left(c_{k}+\delta f\right)-h\left(c_{k}\right)}{\delta f}$ and $f$ is a constant, by (9), the net improvement of the objective function value will be negative when $\delta \rightarrow 0$. The statement follows from the ensuing contradiction.

Property 3. There exists an optimal solution of Problem $(P)$ for which each plant-retailer flow $(i, j)$ is allocated to a single DC, i.e., $x_{i j k}=0$ or $1, \forall i \in I, j \in J, k \in K$, if $h(\cdot)$ is an increasing concave function and $c_{k}$ is unbounded.

Proof: Suppose that there is an optimal solution for Problem $(P)$ with a flow $(i, j)$ that is allocated to at least two different DCs, say $k$ and $l$. Then both $x_{i j k}$ and $x_{i j l}$ are positive. Let us arbitrarily increase $x_{i j k}$ by an amount $\delta=x_{i j l}$ and decrease $x_{i j l}$ to zero. Since $h(\cdot)$ is concave, we obtain,

$$
h\left(c_{k}+\delta f\right)-h\left(c_{k}\right) \leq \delta f \frac{d h\left(c_{k}\right)}{d c_{k}}
$$

and

$$
h\left(c_{l}\right)-h\left(c_{l}-\delta f\right) \geq \delta f \frac{d h\left(c_{l}\right)}{d c_{l}} .
$$

¿From the proof of Lemma 1, we know the net improvement of the objective function value is

$$
\begin{aligned}
& \delta\left(f \frac{h\left(c_{k}+\delta f\right)-h\left(c_{k}\right)}{\delta f}+f_{i j}\left(d\left(i, Y_{k}\right)+d\left(Y_{k}, j\right)\right)-\left(f \frac{h\left(c_{l}+\delta f\right)-h\left(c_{l}\right)}{\delta f}+f_{i j}\left(d\left(i, Y_{l}\right)+d\left(Y_{l}, j\right)\right)\right)\right) \\
& \leq \delta\left(f \frac{d h\left(c_{k}\right)}{d c_{k}}+f_{i j}\left(d\left(i, Y_{k}\right)+d\left(Y_{k}, j\right)\right)\right)-\delta\left(f \frac{d h\left(c_{l}\right)}{d c_{l}}+f_{i j}\left(d\left(i, Y_{l}\right)+d\left(Y_{l}, j\right)\right)\right)=0 .
\end{aligned}
$$

The last equation follows from Lemma 1. Thus, a new feasible solution is obtained which is at least as good as the original optimal solution, and which has one less non-zero variable. 
We repeat the process until each flow is completely allocated to its single DC. The result follows.

If only a single DC needs to be located, by Property 2, the sizing cost term in Problem $(P)$ is a constant in any optimal solution and constraint (3) is redundant. Therefore, the problem becomes

$$
(S P) \quad \min _{\boldsymbol{c}, \boldsymbol{y}} Z^{S P}(\boldsymbol{c}, \boldsymbol{y})=\sum_{i \in I} \sum_{j \in J} \sum_{k \in K} f_{i j}\left(d\left(i, Y_{k}\right)+d\left(Y_{k}, j\right)\right)
$$

Let all origin-destination nodes be the existing nodes. For each existing nodes, its demand is either $\sum_{j \in J} f_{i j}$ (for origin node) or $\sum_{i \in I} f_{i j}$ (for destination node). Thus we obtain the following property:

Property 4. If only a single DC needs to be located, Problem $(P)$ reduces to a standard single facility location problem.

\section{Set covering problem and column generation approach}

In this section, we first reformulate Problem $(P)$ as a set covering problem. Then a column generation approach is presented to solve the problem.

\subsection{Set covering model}

By Property 3, an optimal solution to Problem $(P)$ consists of a partition of origin-destination flow $(i, j)$ into nonempty subsets. We can find an optimal partition from all nonempty subsets of the demand flow set by solving a set covering problem. Let $\mathcal{S}$ be the collection of all nonempty subsets of the origin-destination flow set, i.e., $\mathcal{S}=\left\{W_{1}, W_{2}, \cdots, W_{s}, \cdots\right\}$. Let 
$b_{i j s}$ be a constant that is equal to 1 if origin-destination flow $(i, j)$ is included in subset $W_{s}$ and 0 otherwise, and $o_{s, Y_{k}}$ be the related total cost if $W_{s}$ is assigned to a single DC located at $Y_{k}$. Thus,

$$
o_{s, Y_{k}}=F+\sum_{(i, j) \in W_{s}} f_{i j}\left(d\left(i, Y_{k}\right)+d\left(Y_{k}, j\right)+h\left(\sum_{(i, j) \in W_{s}} f_{i j}\right) .\right.
$$

By Property 4, for a given $W_{s}$, locating the single DC can be reduced to a standard single facility location problem. Suppose that $T_{Y_{k}}^{s}$ is the optimal transportation cost in the standard single facility location problem for $W_{s}$, i.e.,

$$
T_{Y_{k}}^{s}=\min \sum_{(i, j) \in W_{s}} f_{i j}\left(d\left(i, Y_{k}\right)+d\left(Y_{k}, j\right)\right),
$$

and $W_{s}$ is not empty, then we obtain the lowest cost of having one DC serve the flow set $W_{s}$, $o_{s}$, as follows:

$$
\begin{aligned}
o_{s} & =F+T_{Y_{k}}^{s}+h\left(\sum_{(i, j) \in W_{s}} f_{i j}\right) \\
& =F+T_{Y_{k}}^{s}+h\left(\sum_{i \in I} \sum_{j \in J} f_{i j} b_{i j s}\right) .
\end{aligned}
$$

Let decision variable $z_{s}=1$ if the origin-destination flow set $W_{s}$ is selected to be served by a DC and 0 otherwise. Problem $(P)$ or $(E P)$ can be reformulated into a set covering problem as follows:

$$
\begin{array}{r}
(S C) \quad \min _{\boldsymbol{z}} Z^{S C}(\boldsymbol{z})=\sum_{W_{s} \in \mathcal{S}} o_{s} z_{s} \\
\text { subject to } \quad \sum_{W_{s} \in \mathcal{S}} b_{i j s} z_{s} \geq 1, \quad \forall i \in I, j \in J, \\
z_{s} \in\{0,1\}, \quad \forall W_{s} \in \mathcal{S} .
\end{array}
$$

Constraint (12) guarantees that each origin-destination flow belongs to at least one selected origin-destination flow set. Constraint (13) is the integrality constraint. For this set covering problem, we obtain the following property: 
Property 5. There is no optimal solution for Problem $(S C)$ such that $W_{s}$ and $W_{s^{\prime}}$ are assigned to the same DC, where $W_{s}$ and $W_{s^{\prime}}$ are any two origin-destination flow sets.

Proof: Suppose that there is an optimal solution for Problem $(S C)$ such that $W_{s}$ and $W_{s^{\prime}}$ are assigned to the same DC $k$ with location $Y_{k}$. According to the definition of $o_{s}$, we obtain,

$$
\begin{array}{r}
o_{s}+o_{s^{\prime}}=F+T_{Y_{k}}^{s}+h\left(\sum_{i \in I} \sum_{j \in J} f_{i j} b_{i j s}\right) \\
+F+T_{Y_{k}}^{s^{\prime}}+h\left(\sum_{i \in I} \sum_{j \in J} f_{i j} b_{i j s^{\prime}}\right) .
\end{array}
$$

Let $W_{u}=W_{s} \cup W_{s^{\prime}}$, assign $W_{u}$ to DC $k$ and drop $W_{s}$ and $W_{s^{\prime}}$. Then the related cost becomes,

$$
o_{u}=F+T_{Y_{k}}^{s}+T_{Y_{k}}^{s^{\prime}}+h\left(\sum_{i \in I} \sum_{j \in J} f_{i j} b_{i j s}+\sum_{i \in I} \sum_{j \in J} f_{i j} b_{i j s^{\prime}}\right) .
$$

Since $h(x)+h(y) \geq h(x+y)$, for any $x \geq 0$ and $y \geq 0$ (because $h$ is concave), it is easy to see that $o_{u} \leq o_{s}+o_{s^{\prime}}$. By doing this and keeping everything else unchanged, we obtain a new feasible solution, which is at least as good as the old one. The result follows.

This property guarantees that only one origin-destination flow set will be assigned to each generated DC in the optimal solution.

The number of columns involved in this formulation is exponential. Neither the set covering problem nor its linear programming relaxation can be solved by a method that first generates all feasible columns explicitly. We therefore resort to a column generation approach. 


\subsection{Column generation approach}

Let $(\overline{S C})$ be the linear programming relaxation of $(S C)$ and $\left(\overline{S C} C_{\mathcal{S}^{\prime}}\right)$ be the master problem of $(\overline{S C})$ in which a subset $\mathcal{S}^{\prime}$ of $\mathcal{S}$ is available. Thus, the master problem $\left(\overline{S C} \mathcal{S}_{\mathcal{S}^{\prime}}\right)$ is as follows:

$$
\begin{aligned}
& \left(\overline{S C_{\mathcal{S}^{\prime}}}\right) \quad \min _{\boldsymbol{z}} Z^{\overline{S C} \mathcal{S}^{\prime}}(\boldsymbol{z})=\sum_{W_{s} \in \mathcal{S}^{\prime}} o_{s} z_{s} \\
& \text { subject to } \quad \sum_{W_{s} \in \mathcal{S}^{\prime}} b_{i j s} z_{s} \geq 1, \quad \forall i \in I, j \in J, \\
& 0 \leq z_{s} \leq 1 \quad \forall W_{s} \in \mathcal{S}^{\prime}
\end{aligned}
$$

From the theory of linear programming, we know that a solution to a minimization problem is optimal if the reduced cost of each variable is nonnegative. Here, the reduced cost $o_{s}^{*}$ of any subset $W_{s}$ is given by

$$
\begin{gathered}
o_{s}^{*}=o_{s}-\sum_{i \in I} \sum_{j \in J} \lambda_{i j} b_{i j s} \\
=F+T_{Y_{k}}^{s}+h\left(\sum_{i \in I} \sum_{j \in J} f_{i j} b_{i j s}\right)-\sum_{i \in I} \sum_{j \in J} \lambda_{i j} b_{i j s},
\end{gathered}
$$

where $\lambda_{i j}$ is the given value of the dual variable corresponding to constraint (15) for $i$ and $j$. To test whether the current solution is optimal, we determine if there exists a $W_{s}$ with negative reduced cost. Thus the pricing problem is as follows:

$$
\begin{aligned}
& (P P) \quad \min \sum_{i \in I} \sum_{j \in J} f_{i j}\left(d\left(i, Y_{k}\right)+d\left(Y_{k}, j\right)\right) x_{i j k}+h\left(\sum_{i \in I} \sum_{j \in J} f_{i j} x_{i j k}\right)-\sum_{i \in I} \sum_{j \in J} \lambda_{i j} x_{i j k}, \\
& \text { subject to } \quad x_{i j k} \in\{0,1\}, Y_{k} \in R^{2}, \quad \forall i \in I, j \in J, k \in K .
\end{aligned}
$$

Here, we drop the constant $F$ and will add it to the optimal objective function value after the pricing problem is solved. The pricing problem is a mixed integer nonlinear programming 
problem. The difficulty to solve this problem is two fold. First, the DC location $Y_{k}$ is a variable. Therefore, $\sum_{i \in I} \sum_{j \in J} f_{i j}\left(d\left(i, Y_{k}\right)+d\left(Y_{k}, j\right)\right) x_{i j k}$ is a mixed integer nonlinear term. Second, the function $h(\cdot)$ is a concave function. However, according to Property 1, we know that DCs will be located at grid points in an optimal solution. Thus, we choose the DC locations from the grid points. For given grid point, $\sum_{i \in I} \sum_{j \in J} f_{i j}\left(d\left(i, Y_{k}\right)+d\left(Y_{k}, j\right)\right) x_{i j k}$ is a linear function of $x_{i j k}$. If for every grid point we have nonnegative reduced cost, then every subset $W_{s}$ has nonnegative reduced cost. Let $G$ be the set of all grid points. The pricing problem reduces to the following problem $\left(R P P_{k}\right)$ for each grid point $Y_{k} \in G$ :

$$
\begin{aligned}
& \left(R P P_{k}\right) \quad \min _{\boldsymbol{x}} Z^{R P P_{k}}(\boldsymbol{x})=\sum_{i \in I} \sum_{j \in J}\left(f_{i j} d_{i j k}-\lambda_{i j}\right) x_{i j k}+h\left(\sum_{i \in I} \sum_{j \in J} f_{i j} x_{i j k}\right), \\
& \text { subject to } \quad x_{i j k} \in\{0,1\}, \quad \forall i \in I, j \in J
\end{aligned}
$$

where $d_{i j k}=d\left(i, Y_{k}\right)+d\left(Y_{k}, j\right)$ is a known constant here since $Y_{k}$ is a grid point. Coincidently, the pricing problem is very similar to the one in Shen, Coullard, and Daskin (2003). They developed an effective approach to solve such the pricing problem. Following their idea, we can develop an algorithm to solve the pricing problems in polynomial time.

Let $\boldsymbol{x}^{*}$ be an optimal solution to Problem $\left(R P P_{k}\right)$ and the minimum reduced-cost set $W_{s}=\left\{(i, j): x_{i j k}^{*}=1\right\}$, and $G_{k}^{*}=F+Z^{R P P_{k}}\left(\boldsymbol{x}^{*}\right)$. If $G_{k}^{*} \geq 0$, then we can conclude that there is no set $W_{s}$ assigning to grid point $k$ with negative reduced cost. If $\forall k, G_{k}^{*} \geq 0$, we can conclude that there is no set $W_{s} \in \mathcal{S}$ with negative reduced cost.

Now suppose that all flows $(i, j), \forall i \in I, j \in J$, have been sorted so that:

$$
\frac{f_{i_{1} j_{1}} d_{i_{1} j_{1} k}-\lambda_{i_{1} j_{1}}}{f_{i_{1} j_{1}}} \leq \frac{f_{i_{2} j_{2}} d_{i_{2} j_{2} k}-\lambda_{i_{2} j_{2}}}{f_{i_{2} j_{2}}} \leq \cdots \leq \frac{f_{i_{\gamma} j_{\gamma}} d_{i_{\gamma} j_{\gamma} k}-\lambda_{i_{\gamma} j_{\gamma}}}{f_{i_{\gamma} j_{\gamma}}}
$$


where $\gamma$ is the total number of non-zero flows. Then, the following theorem is an immediate consequence from Shen, Coullard, and Daskin (2003).

Theorem 1. There is an optimal solution $x_{i j k}^{*}$ to Problem $\left(R P P_{k}\right)$ in which the following properties hold:

1. If $f_{i j} d_{i j k} \geq \lambda_{i j}$, then $x_{i j k}^{*}=0, \forall i \in I, j \in J$.

2. If $x_{i_{t} j_{t} k}^{*}=1$, for some $t \in\{1,2, \cdots, \gamma\}$, then $x_{i_{l} j_{l} k}^{*}=1$, for all $l \in\{1,2, \cdots, t-1\}$.

Using Theorem 1, we can develop an algorithm to solve the pricing problems in polynomial time. In fact, we can solve Problem $\left(R P P_{k}\right)$ by enumeration, i.e., by generating all solutions with the properties and selecting the one with the lowest objective function value. The complexity of the pricing problem is $O\left((m+n)^{2} m n \log (m n)\right)$, where $m$ and $n$ are total numbers of plant and retailer nodes, respectively.

\subsection{Greedy drop heuristic algorithm}

In this section we develop a greedy drop heuristic algorithm to solve Problem $(P)$. The purpose of this section is twofold. First, we need a set of initial columns. Second, we need a good upper bound. Both objectives can be achieved by using a greedy drop heuristic algorithm. To present this algorithm we first need to introduce the concept of a shortest path flow set.

\subsubsection{The shortest path flow set}

Before we develop the heuristic algorithm, we need to introduce a shortest path flow set of each grid point for a given grid construction. The grid construction under the Manhattan 
distance in this paper is defined in Section 2.

We define the shortest path flow set for each grid point as follows. Let $(i, j)$ refer to a pair of plant and retailer with coordinates $\left(x_{i}, y_{i}\right)$ and $\left(x_{j}, y_{j}\right)$, respectively, and $s$ refer to any grid point with location coordinates $\left(x_{s}, y_{s}\right)$. Then the shortest path flow set of grid point $s$, $P_{s}$, is given by:

$$
\begin{aligned}
& P_{s}:=\left\{(i, j): x_{i} \leq x_{s}, y_{i} \geq y_{s}, x_{j} \geq x_{s}, y_{j} \leq y_{s}\right\} \cup\left\{(i, j): x_{i} \leq x_{s}, y_{i} \leq y_{s}, x_{j} \geq x_{s}, y_{j} \geq y_{s}\right\} \\
& \cup\left\{(i, j): x_{i} \geq x_{s}, y_{i} \geq y_{s}, x_{j} \leq x_{s}, y_{j} \leq y_{s}\right\} \cup\left\{(i, j): x_{i} \geq x_{s}, y_{i} \leq y_{s}, x_{j} \leq x_{s}, y_{j} \geq y_{s}\right\} .
\end{aligned}
$$

Under the Manhattan distance, if we select $s$ as a DC location, then for all $(i, j) \in P_{s}$ the shortest path from plant $i$ to retailer $j$ passes through $s$. Figure 2 provides examples of sets $P_{s}$.

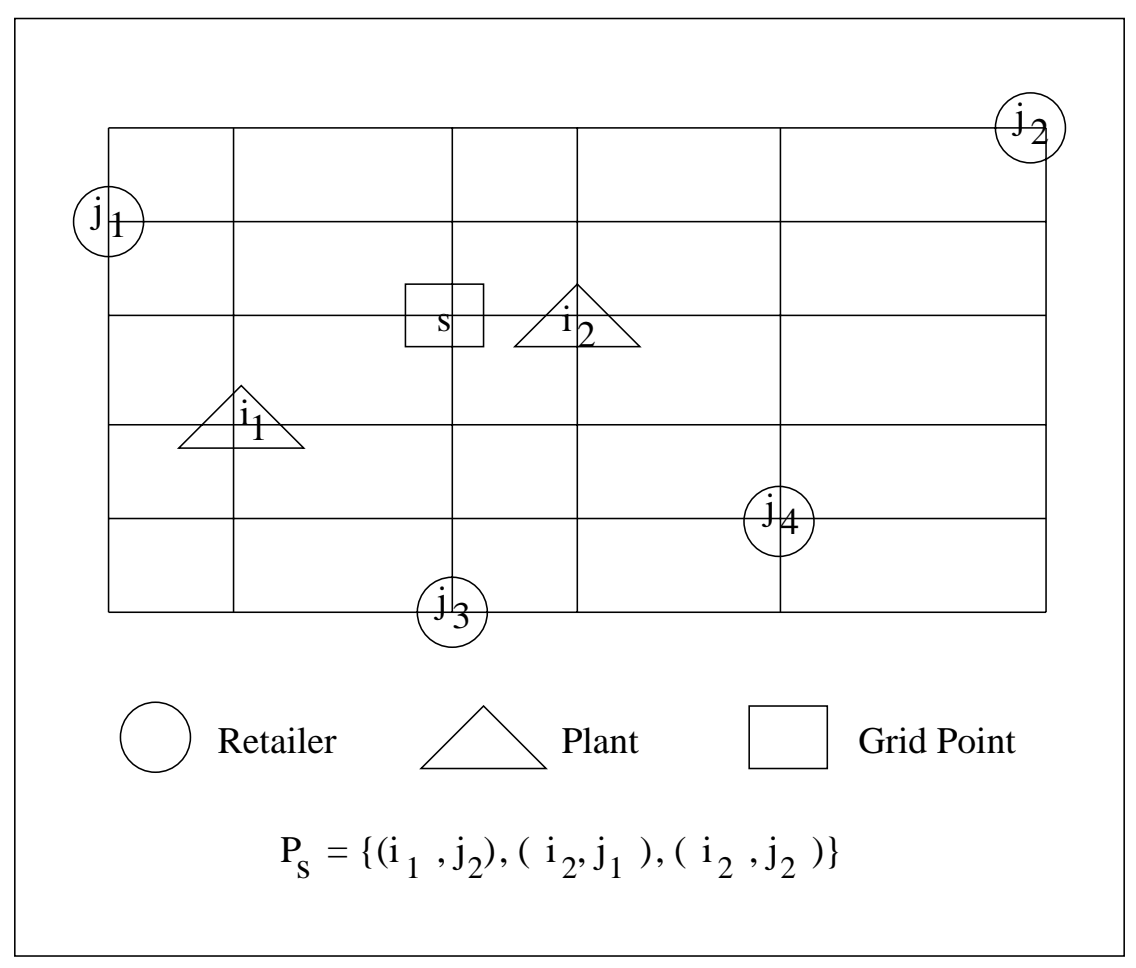

Figure 2: An illustration of the shortest path flow set 


\subsubsection{Greedy drop heuristic algorithm}

The greedy drop heuristic algorithm is built based on the shortest path flow set of each grid point. Let $f_{s}=\sum_{(i, j) \in P_{s}} f_{i j}$. Then $f_{s}$ is the total flow amount that passes through $s$ with the shortest distance. Intuitively, the larger $f_{s}$ is, the more important the grid point $s$ is because of the concave sizing cost function. Thus, the basic idea for this heuristic algorithm is to use the order of $f_{s}$ to find a heuristic solution.

The greedy drop heuristic algorithm is as follows: First, we define $W$ to be the set of all plant-retailer pairs, $(i, j), \forall i \in I, j \in J$, and $W^{u}$ to be the set of $(i, j)$ that are currently not assigned to any DC. Initialize $G=\{s: s$ is a grid point $\}$ and $W^{u}:=W$. Find the shortest path flow set, $f_{s}=\sum_{(i, j) \in P_{s} \cap W^{u}} f_{i j}, \forall s \in G$. Pick the grid point $s$ with the largest $f_{s}$ value as a candidate DC location and assign all $(i, j) \in P_{s} \cap W^{u}$ to this DC, and let $W_{s}=\left\{(i, j) \in P_{s} \cap W^{u}\right\}$. Update $W^{u}:=W^{u} \backslash P_{s}$ and $G:=G \backslash\{s\}$, and repeat the procedure until $W^{u}$ is empty. Using this procedure, we obtain a collection of initial $W_{s}$ sets and their corresponding grid points.

Second, we drop one initial $W_{s}$, assign the flows of this set to the nearest available grid point that is selected above, and recalculate the objective function. The procedure is repeated for all initial $W_{s}$ sets. Then we drop the $W_{s}$ with the most improved objective function, update $W_{s}$ and repeat the procedure until we see no further improvement in the objective function value.

Each step of the algorithm generates a collection of $W_{s}$ sets. All of them could be used as an initial subset $\mathcal{S}^{\prime}$ in the column generation algorithm. We also note that the solution of the greedy drop heuristic algorithm provides an upper bound for the original problem $(P)$. 


\subsection{Column generation algorithm}

Now, we are ready to give a step-wise description of the column generation algorithm as follows:

1. Generate the initial subset $\mathcal{S}^{\prime}$ as described above.

2. Solve the linear programming relaxation to obtain the vector of current dual costs.

3. Use the pricing algorithm to determine if there is subset $W_{s}$ whose associate column has negative reduced cost. If such subset $W_{s}$ exist, then add it to $\mathcal{S}^{\prime}$ and go to step 2.

4. If no such subset $W_{s}$ exist, then stop. Solve the set covering problem $(S C)$ for $\mathcal{S}^{\prime}$.

\section{Computational Results}

In this section, we test the performance of the greedy drop heuristic algorithm and the column generation algorithm. The efficiency of the algorithms are tested by solving randomly generated problems of different sizes. The algorithms were programmed in Microsoft Visual $\mathrm{C}++5.0$ and CPLEX8.1. All of the experimental tests were carried out on a Dell OptiPlex GX240 with 512MB RAM and 1.8GHz CPU. Computation times are in seconds.

\subsection{Data generation}

First, we generated locations of each plant and retailer, which are given by their $x$ and $y$ coordinates. These coordinate values were randomly selected from $U(0,200)$, where $U$ denotes a uniform distribution. For each pair of plant-retailer, the amount of flow was 
randomly drawn from $U(5,30)$. The constant fixed charge cost of a DC, $F$, was 1000 . Also, the DC sizing cost function was set to be $h\left(c_{k}\right)=50 \sqrt{c_{k}}$. The sizes of randomly generated problems were the combination of the following parameters: number of plant nodes $m=5,10$; number of retailer nodes $n=10,15,20,25,30$; and total number of flows from plants to retailers were 50,60, 70, 80, 90, 100 .

\subsection{Computational performance}

Here we report the performance of the greedy drop heuristic algorithm and the column generation algorithm for Problem $(P)$.

We had two schemes for adding the negative reduced cost column to the linear program after having solved the pricing algorithm. In the first one (called $C g 1$ ), we iteratively added a column with the smallest negative reduced cost from all $k \in G$ to the linear program. We continued the procedure until the pricing problems could not find a negative reduced cost column, i.e., the linear programming relaxation problem was solved optimally. In the second scheme (called $C g^{2}$ ), we remove some columns during the column generation procedure so as to reduce the size of the restricted master problem. The criterion of removing a column is as follows: calculate the average reduced cost for the existing columns that have positive reduced costs, then remove those columns whose reduced cost is greater than or equal to two times of the average reduced cost.

Table 1 summarizes the computational results for the greedy drop heuristic algorithm and the column generation algorithms (both $C g 1$ and $C g 2$ ). Some headers of the columns in Table 1 are: 
- CPU time (seconds): Total CPU time (seconds) consumed for solving the instance;

- Greedy: greedy drop algorithm presented in Section 3.3;

- Greedy/lb: (greedy solution value - linear relaxation lower bound)/linear relaxation lower bound * 100;

- Cg1/lb: (Scheme $(C g 1)$ solution value - linear relaxation lower bound)/linear relaxation lower bound * 100;

- Cg2/lb: (Scheme (Cg2) solution value - linear relaxation lower bound)/linear relaxation lower bound * 100;

¿From Table 1, we can see that both greedy drop and column generation with scheme $C g 2$ algorithms performed extremely well for the given problem sizes, in terms of computational time and heuristic gap. In fact, they have the same heuristic gaps for the given instances. The column generation algorithm with scheme $C g 1$ also performed well, except for the last problem instance. For this instance, we forced the integer programming solver to stop after one hour. Therefore, the heuristic gap for this case is large, compared with others. However, the column generation algorithm with scheme $C g 1$ solved the problem optimally for eight instances.

We also tested very large size problems with hundreds of retailers and thousands of flows. For both schemes, the column generation algorithm could not generate a solution. But the increased problem size did not affect the greedy drop heuristic algorithm as expected, in terms of computational time. 
Table 1: Computational Results

\begin{tabular}{|c|c|c|rrr|ccc|}
\hline \multirow{2}{*}{$m$} & \multirow{2}{*}{$n$} & \multirow{2}{*}{ Flow Number } & \multicolumn{2}{|c|}{ CPU time (seconds) } & \multicolumn{4}{|c|}{ Gap (\%) } \\
\cline { 4 - 9 } & & Greedy & Cg1 & Cg2 & Greedy/lb & Cg1/lb & Cg2/lb \\
\hline 5 & 10 & 50 & 0 & 30.162 & 7.844 & 0.002 & 0.000 & 0.002 \\
5 & 15 & 60 & 0 & 90.068 & 17.562 & 0.003 & 0.000 & 0.003 \\
5 & 20 & 70 & 0 & 134.529 & 15.563 & 0.014 & 0.000 & 0.014 \\
5 & 25 & 80 & 0.016 & 210.166 & 29.72 & 0.009 & 0.000 & 0.009 \\
5 & 30 & 90 & 0.016 & 593.015 & 87.658 & 0.032 & 0.000 & 0.032 \\
10 & 10 & 60 & 0 & 519.898 & 15.953 & 0.587 & 0.210 & 0.587 \\
10 & 15 & 70 & 0.047 & 73.682 & 15.344 & 0.664 & 0.000 & 0.664 \\
10 & 20 & 80 & 0.016 & 162.329 & 38.251 & 0.666 & 0.000 & 0.666 \\
10 & 25 & 90 & 0.015 & 270.556 & 50.157 & 0.355 & 0.000 & 0.355 \\
10 & 30 & 100 & 0.032 & $3886.83^{*}$ & 52.58 & 0.845 & $8.783^{*}$ & 0.845 \\
\hline
\end{tabular}




\section{Conclusion}

This paper proposes a model for studying the problem of simultaneous consideration of siting and sizing of DCs on the plane. The problem minimizes the total transportation cost and DC sizing costs, which include its fixed charge cost and concave sizing cost. The most significant results are the grid node optimality property under the Manhattan distance and full flow assignment property under the concave sizing cost assumption. These results allow us to reformulate the problem as a set covering problem and develop an efficient greedy drop heuristic algorithm and a column generation heuristic algorithm. The computational results show the efficiency and accuracy of the algorithms.

Several extensions of the problem are possible. For example, we assume that the fixed charge sizing cost of a DC is constant for all potential DC sites. Developing a model for location related fixed charge sizing cost is a possible topic for further research. The problem could also be investigated under different distance measures, for example, the Euclidean distance. The solution approach developed in this paper can also be extended to network location and sizing problem, where DCs have to be located on a given network.

\section{Acknowledgments}

The authors would like to acknowledge support from the National Science Foundation via Grant No. DMI-0300370. They would also like to thank the referees for their constructive comments. 


\section{References}

Aboolian, R., O. Berman, and D. Krass (2004). Competitive facilities location and design problems on a network. Presented at the EURO XX Conference in Rhodes, Greece, July 2004.

Brimberg, J. and R. F. Love (1994). A location problem with economies of scale. Studies in Locational Analysis 7, 9-19.

Brimberg, J. and A. Mehrez (1997). A note on the allocation of queueing facilities using a minisum criterion. Journal of the Operational Research Society 48, 195-201.

Brimberg, J., A. Mehrez, and G. O. Wesolowsky (1997). Allocation of queueing facilities using a minimax criterion. Location Science 5, 89-101.

Cooper, L. (1963). Location-allocation problems. Operations Research 11, 331-343.

Cooper, L. (1964). Heuristic methods for location-allocation problems. SIAM Review 6, $37-53$.

Cooper, L. (1967). Solutions of generalized locational equilibrium models. Journal of Regional Science \%, 1-18.

Cormier, G. and E. A. Gunn (1996a). On the coordination of warehouse sizing, leasing and inventory policy. IIE Transactions 28, 149-154.

Cormier, G. and E. A. Gunn (1996b). Simple models and insights for warehouse sizing. Journal of the Operational Research Society 47, 690-696.

Eilon, S., C. D. T. Watson-Gandy, and N. Christofides (1971). Distribution Management: Mathematical Modelling and Practical Analysis. Hafner, New York. 
Heragu, S. (1997). Facilities Design. PWS Publishing Company.

Hodgson, M. J., K. E. Rosing, and F. Shmulevitz (1993). A review of location-allocation applications literature. Studies in Locational Analysis 5, 2-29.

Huang, S., R. Batta, K. Klamroth, and R. Nagi (2005). The $K$-connection location problem in a plane. Annals of Operations Research 136, 193-209.

Jucker, J. V., R. C. Carlson, and D. H. Kropp (1982). The simultaneous determination of plant and leased warehouse capacities for a firm facing uncertain demand in several regions. IIE Transactions 14, 99-108.

Krarup, J. and P. M. Pruzan (1990). Ingredients of locational analysis. In P. B. Mirchandani and R. L. Francis (Eds.), Discrete Location Theory, pp. 1-54. John Wiley, New York.

Lee, C. Y. (1991). An optimal algorithm for the multiproduct capacitated facility location problem with a choice of facility type. Computers and Operations Research 20, 527540.

Lowe, T. J., R. L. Francis, and E. W. Reinhardt (1979). A greedy network flow algorithm for a warehouse leasing problem. AIIE Transactions 11, 170-182.

Mazzola, J. B. and A. W. Neebe (1999). Lagrangian-relaxation-based solution procedures for a multiproduct capacitated facility location problem with choice of facility type. European Journal of Operational Research 115, 285-299.

Shen, Z. M., C. Coullard, and M. S. Daskin (2003). A joint location-inventory model. Transportation Science 37, 40-55. 
Sung, C. S. and Y. H. Han (1992). Determination of automated storage/retrieval system size. Engineering Optimization 19, 269-286.

White, J. A. and R. L. Francis (1971). Normative models for some warehouse sizing problems. AIIE Transactions 3, 185-190. 